\title{
International Scientific Collaboration Is Needed to Bridge Science to Society: USERN2020 Consensus Statement
}

\author{
Sara Momtazmanesh ${ }^{1,2,3}$ - Amene Saghazadeh ${ }^{1,2,3}$ - Juan Carlos Aldave Becerra ${ }^{1,4} \cdot$ Kiarash Aramesh $^{1,5}$. \\ Francisco J. Barba ${ }^{1,6} \cdot$ Federico Bella $^{1,7}$. Anna Blakney ${ }^{1,8}$ - Massimo Capaccioli ${ }^{1,9} \cdot$ Rossella Castagna ${ }^{1,10,11}$. \\ Umberto Crisanti ${ }^{1,12} \cdot$ Tigran Davtyan ${ }^{1,13} \cdot$ Tommaso Dorigo $^{1,14}$. Julie Ealy ${ }^{1,15}$. Mehdi Farokhnia ${ }^{1,16}$. \\ Giulia Grancini $^{1,17}$ - Manoj Gupta ${ }^{1,18}$. Amine Harbi ${ }^{1,19}$ - Wojciech Krysztofiak ${ }^{1,20}$. Arutha Kulasinghe ${ }^{1,21} \cdot$ Chi- $^{1,24}$ \\ Ming Lam ${ }^{1,22}$. Alexander Leemans ${ }^{1,22} \cdot$ Brian Lighthill $^{1,23}$. Vittorio Limongelli ${ }^{1,24,25}$. Paola Lopreiato ${ }^{1,26}$. \\ Livio Luongo ${ }^{1,27}$. Christopher Ryan Maboloc ${ }^{1,28} \cdot$ Reza Malekzadeh $^{1,29}$. Orlando Costa Gomes ${ }^{1,30}$. \\ Milos Milosevic $^{1,31}$. Jan Nouwen ${ }^{1,32}$. Delfín Ortega-Sánchez ${ }^{1,33} \cdot$ John Pawelek ${ }^{1,34}$. Surapati Pramanik ${ }^{1,35}$. \\ Seeram Ramakrishna ${ }^{1,36}$. Ortwin Renn ${ }^{1,37}$. Serena Sanseviero ${ }^{1,38}$ - Daniel Sauter ${ }^{1,39}$. Michael Schreiber ${ }^{1,40}$. \\ Frank W. Sellke ${ }^{1,41} \cdot$ Mohammad-Ali Shahbazi $^{1,42} \cdot$ Natalya Shelkovaya $^{1,43} \cdot$ Wayne H. Slater ${ }^{1,44}$. Didier Snoeck ${ }^{1,45}$. \\ Slawomir Sztajer ${ }^{1,46} \cdot$ Lucina Q. Uddin $^{1,47} \cdot$ Liz Veramendi-Espinoza $^{1,48} \cdot$ Ricardo Vinuesa $^{1,49} \cdot$ Walter C. Willett $^{1,50}$. \\ Dongrui Wu ${ }^{1,51} \cdot$ Karolina Żyniewicz ${ }^{1,52} \cdot$ Nima Rezaei ${ }^{1,2,3}$.
}

Accepted: 7 April 2021 / Published online: 11 May 2021

(C) The Author(s), under exclusive licence to Springer Nature Switzerland AG 2021

\begin{abstract}
Scientific collaboration has been a critical aspect of the development of all fields of science, particularly clinical medicine. It is well understood that myriads of benefits can be yielded by interdisciplinary and international collaboration. For instance, our rapidly growing knowledge on COVID-19 and vaccine development could not be attained without expanded collaborative activities. However, achieving fruitful results requires mastering specific tactics in collaborative efforts. These activities can enhance our knowledge, which ultimately benefits society. In addition to tackling the issue of the invisible border between different countries, institutes, and disciplines, the border between the scientific community and society needs to be addressed as well. International and transdisciplinary approaches can potentially be the best solution for bridging science and society. The Universal Scientific Education and Research Network (USERN) is a non-governmental, non-profit organization and network to promote professional, scientific research and education worldwide. The fifth annual congress of USERN was held in Tehran, Iran, in a hybrid manner on November 7-10, 2020, with key aims of bridging science to society and facilitating borderless science. Among speakers of the congress, a group of top scientists unanimously agreed on The USERN 2020 consensus, which is drafted with the goal of connecting society with scientific scholars and facilitating international and interdisciplinary scientific activities in all fields, including clinical medicine.
\end{abstract}

Keywords International $\cdot$ Collaboration $\cdot$ Science $\cdot$ Society $\cdot$ Research $\cdot$ Policy

All authors are affiliated to Universal Scientific Education and Research Network (USERN), The World.

This article is part of the Topical Collection on Covid-19

Nima Rezaei

Extended author information available on the last page of the article

\section{Introduction}

Scientific collaboration has been a critical aspect of the development of all fields of science, including clinical medicine, from centuries ago; nonetheless, in recent decades, it has even drawn more attention from the academic society [1,2]. Collaboration is defined as "the act of working with another person or group of people to create or produce something" by the Oxford English Dictionary. The core concept is achieving a common goal, which is usually to advance scientific knowledge by harmonizing knowledge, skills, tools, and resources [3, 4]. 
Historically, scientific research evolved through four ages: the individual, the institutional, the national, and the international [5]. Before the twentieth century, scientific efforts were mostly individual activities, and most of the scholars were working alone, which resulted in a scarce number of papers with more than one author. However, in many cases, the discoveries were not made solo, and the role of other contributors was less acknowledged in this approach [1]. From the beginning of the twentieth century, institutional and national research collaboration flourished. In recent decades, scientific research has entered its fourth era, and we are witnessing substantial growth in international collaboration [6]. In 2012, approximately a quarter of the papers indexed in the Web of Science were produced as a result of international collaboration [7].

Life sciences, including clinical medicine, are an outstanding showcase for internationally collaborative scientific efforts. A quintessential example of collaborative activities is the investigation of emerging viruses, especially SARSCoV-2 (COVID-19). Without collaborative measures, such as the development of international registries and sharing expertise as well as technologies, we could not obtain our current rapidly growing understanding of COVID-19 [8-10]. International collaboration plays a major role in accelerating and improving vaccine development and assessment processes as well [11].

\section{Why and How to Conduct International Collaboration?}

Collaboration and internationalism can help researchers have access to expert scientists in their field, additional resources, and equipment or funds, learn different methods, gain additional knowledge, enhance productivity, and increase the visibility of their work. Borderless scientific collaboration, especially when it is formed as a mentoring program, can help substantially in nourishing and education of young students. Not only does international collaboration increase scientific achievements quantitatively, but it can also enhance our understanding of knowledge qualitatively. Many findings that are obtained through multinational research activities were impossible to attain without working together. Borderless collaboration helps us find answers for complex problems, which may seem insurmountable if we want to tackle them solo [1, 3, 12].

When we choose to pursue international collaborations, mastering the tactics for achieving fruitful results is critical. First, to have an effective collaboration, the work style of the collaborative partners should fit. That may be why many researchers choose to work with people they know and trust and why many collaborative projects start from informal interactions [12]. Second, in a successful collaboration, partners need to communicate with each other effectively. Roles, responsibilities, and the credit collaborators receive for the final product of collaboration should be stated clearly. An efficient collaboration also requires competent management, which can assign each collaborator the proper task and ensure that each collaborator meets the expectations and receives what they expect from the team.

To maintain such a fruitful project, collaborators should also avoid some actions. Many challenges to collaborative science may arise from personality differences and collaborators' urge to control their peers [13]. Additionally, we need to pay attention to avoid any collaborator's exploitation and having ghost authors in large-scale international collaborations.

However, doubtlessly, several factors may hinder international collaboration [14]. A recent study cited lack of funds, limitation in the dissemination of data, bias against researchers from specific countries (particularly developing countries), and various academic standards worldwide as the most common obstructions of international scientific collaboration [15].

\section{Science and Society}

In addition to tackling the issue of the invisible border between different countries and institutes, the border between the scientific community and society needs to be addressed as well. Bridging science and society is crucial since the ultimate aim of science is to serve society in overcoming challenges such as climate changes, food scarcity, and biodiversity loss. The urge for facilitating communication between the scientific community and society should be highlighted. The scientific community should promote science in an enticing and easy to comprehend manner and provide opportunities for society to engage in scientific activities. Scientists should also be aware of the key societal challenges in their field so that they can find suitable solutions for them. The COVID-19 pandemic highlighted the crucial need for bridging the scientific community and society in clinical medicine [16].

\section{USERN2020 Consensus Statement}

Universal Scientific Education and Research Network (USERN) is a non-governmental, non-profit organization and network, which was established on January 1, 2016, to promote professional, scientific research and education worldwide [17-20]. In 2020, the USERN established the U100 platform, including a network of at least one-hundred academic institutes worldwide with the vision of providing an ideal environment for reciprocal scientific exchange all around the world by removing the borders between countries and disciplines [21].

The fifth annual congress of USERN was held in Tehran, Iran, in a hybrid manner [22] on November 7-10, 2020, with 
Table 1 The USERN2020 consensus
1 Committing to principles of conducting a fruitful scientific collaboration, such as effective and clear communication, competent management, and properly giving credit to each collaborator for their efforts in all collaborative works is an essential part of scientific collaboration

2 Any bias based on race, nationality, gender, and academic position should be avoided in all collaborative works

3 Organizing programs making science enticing to the public and teaching young scientists how to explain science in a non-scientific language play a critical role in connecting science and society

4 The USERN must facilitate mutual sharing of information and technology to support research and educational activities yielding mutual benefits, which can result in new collaborative scientific production

5 The USERN must facilitate share of expertise and providing opportunities for education of young researchers and scientists in a format of providing opportunities for cooperation of senior and junior researchers in scientific projects, holding workshops and schools, and exchange of researchers and students

6 The USERN must facilitate planning to translate the science to understandable materials for the public and make the application of science to society the overall aims of bridging science to society and facilitating borderless science. Forty-eight of the speakers, including some of top 1 percent of scientists in the world, who were from fifteen countries, unanimously agreed on The USERN 2020 consensus, which is drafted with the goal of connecting society with scientific scholars and facilitating international and interdisciplinary scientific activities. Table 1 demonstrates The USERN 2020 Consensus, which is comprised of 6 articles.

\section{Conclusion}

The USERN is determined to promote and facilitate international scientific collaboration and continues its efforts on its way to remove the borders between different institutions, nations, and disciplines and between the scientific community and the society to achieve its ultimate goal, which is borderless science in all scientific fields, including clinical medicine, which is an outstanding showcase for internationally collaborative scientific efforts.

Author Contribution SM drafted the manuscript. NR designed and supervised the project. All authors read and critically revised the manuscript.

Availability of Data and Material Not applicable.

Code Availability Not applicable.

\section{Declarations}

Ethics Approval Not applicable.

Consent to Participate Not applicable.
Consent for Publication Not applicable.

Conflict of Interest The authors declare no competing interests.

\section{References}

1. Beaver DD, Rosen R. Studies in scientific collaboration. Scientometrics. 1978;1(1):65-84.

2. Adams J. The rise of research networks. Nature. 2012;490(7420): $335-6$.

3. Katz JS, Martin BR. What is research collaboration? Res Policy. 1997;26(1):1-18.

4. Olechnicka A, Ploszaj A, Celińska-Janowicz D. The Geography of Scientific Collaboration (1st ed.). Routledge. 2018. https://doi.org/ 10.4324/9781315471938.

5. Adams J. The fourth age of research. Nature. 2013;497(7451):55760.

6. Coccia M, Bozeman B. Allometric models to measure and analyze the evolution of international research collaboration. Scientometrics. 2016;108(3):1065-84.

7. Mindeli LE, Markusova VA. Bibliometric studies of scientific collaboration: international trends. Autom Doc Math Ling. 2015;49(2):59-64.

8. Momtazmanesh S, Ochs HD, Uddin LQ, Perc M, Routes JM, Vieira DN, et al. All together to fight COVID-19. Am J Trop Med Hyg. 2020;102(6):1181-3.

9. Mohamed K, Rodriguez-Roman E, Rahmani F, Zhang H, Ivanovska M, Makka SA, et al. Borderless collaboration is needed for COVID-19-A disease that knows no borders. Infect Control Hosp Epidemiol. 2020;41(10):1245-6.

10. Collins A, Florin M-V, Renn O. COVID-19 risk governance: drivers, responses and lessons to be learned. J Risk Res. 2020;23(7-8):1073-82.

11. Rappuoli R, Hanon E. Sustainable vaccine development: a vaccine manufacturer's perspective. Curr Opin Immunol. 2018;53:111-8.

12. Bozeman B, Corley E. Scientists' collaboration strategies: implications for scientific and technical human capital. Res Policy. 2004;33(4):599-616.

13. Bozeman B, Gaughan M, Youtie J, Slade CP, Rimes H. Research collaboration experiences, good and bad: dispatches from the front lines. Sci Public Policy. 2016;43(2):226-44. 
14. See M. 18 International collaboration: are the challenges worth the benefits? J Anim Sci. 2018;96(suppl_3):2.

15. Lund BD. Is academic research and publishing still leaving developing countries behind?. Account Res. 2021;0:0:1-9. https://doi. org/10.1080/08989621.2020.1774373.

16. Richard A, Roehrl WL, Mukherjee S. The COVID-19 pandemic: a wake-up call for better cooperation at the science-policy-society interface. In: Desa U, editor. POLICY BRIEF NO62; 2020. URL: https://www.un.org/development/desa/dpad/publication/un-desapolicy-brief-62-the-covid-19-pandemic-awake-up-call-for-bettercooperation-at-the-science-policy-society-interface/.

17. Rezaei N. Universal Scientific Education and Research Network (USERN):a new horizon for science. Acta Med Iran. 2016;54(1): $1-3$.

18. Rezaei N. Universal Scientific Education and Research Network (USERN): to make the knowledge without borders. Acta Med Iran. 2017;55(1):1-5.

19. Rezaei N. Universal Scientific Education and Research Network (USERN): twinkling stars unite to make the world glow. Acta Med Iran. 2018;56(1):1-3.
20. Rahmani FK-FM, Hanaei S, Aminorroaya A, Delavari F, ParyadZanjani S, Sadat Ahmadi N, et al. Universal Scientific Education and Research Network (USERN): step strong in scientific networking. Acta Med Iran. 2019;57(1):1-4.

21. Momtazmanesh S, Ochs HD, Uddin LQ, Perc M, Routes JM, Vieira DN, et al. All together to Fight COVID-19. Am J Trop Med Hyg. 2020;102(6):1181-1183. https://doi.org/10.4269/ajtmh. 20-0281.

22. Hanaei S, Takian A, Majdzadeh R, Maboloc CR, Grossmann I, Gomes $\mathrm{O}$, et al. Emerging standards and the hybrid model for organizing scientific events during and after the COVID-19 pandemic. Disaster Med Public Health Prep. 2020:1-17. https://doi.org/10. 1017/dmp.2020.406.

Publisher's Note Springer Nature remains neutral with regard to jurisdictional claims in published maps and institutional affiliations.

\section{Affiliations}

Sara Momtazmanesh ${ }^{1,2,3} \cdot$ Amene Saghazadeh ${ }^{1,2,3} \cdot$ Juan Carlos Aldave Becerra ${ }^{1,4} \cdot$ Kiarash Aramesh $^{1,5}$. Francisco J. Barba ${ }^{1,6}$. Federico Bella ${ }^{1,7}$. Anna Blakney ${ }^{1,8}$ - Massimo Capaccioli ${ }^{1,9}$ • Rossella Castagna ${ }^{1,10,11}$. Umberto Crisanti ${ }^{1,12} \cdot$ Tigran Davtyan $^{1,13} \cdot$ Tommaso Dorigo $^{1,14}$. Julie Ealy ${ }^{1,15}$. Mehdi Farokhnia ${ }^{1,16}$. Giulia Grancini $^{1,17}$. Manoj Gupta ${ }^{1,18}$. Amine Harbi ${ }^{1,19}$. Wojciech Krysztofiak ${ }^{1,20}$. Arutha Kulasinghe ${ }^{1,21}$. ChiMing Lam ${ }^{1,22}$ - Alexander Leemans ${ }^{1,22} \cdot$ Brian Lighthill $^{1,23} \cdot$ Vittorio Limongelli $^{1,24,25}$. Paola Lopreiato ${ }^{1,26}$. Livio Luongo ${ }^{1,27}$. Christopher Ryan Maboloc ${ }^{1,28} \cdot$ Reza Malekzadeh $^{1,29}$. Orlando Costa Gomes ${ }^{1,30}$. Milos Milosevic $^{1,31}$ • Jan Nouwen ${ }^{1,32}$. Delfín Ortega-Sánchez ${ }^{1,33}$. John Pawelek ${ }^{1,34}$. Surapati Pramanik ${ }^{1,35}$. Seeram Ramakrishna ${ }^{1,36}$. Ortwin Renn ${ }^{1,37}$. Serena Sanseviero ${ }^{1,38}$. Daniel Sauter ${ }^{1,39}$. Michael Schreiber ${ }^{1,40}$. Frank W. Sellke ${ }^{1,41} \cdot$ Mohammad-Ali Shahbazi $^{1,42} \cdot$ Natalya Shelkovaya $^{1,43}$. Wayne H. Slater ${ }^{1,44}$. Didier Snoeck ${ }^{1,45}$. Slawomir Sztajer ${ }^{1,46} \cdot$ Lucina Q. Uddin $^{1,47} \cdot$ Liz Veramendi-Espinoza $^{1,48} \cdot$ Ricardo Vinuesa $^{1,49} \cdot$ Walter C. Willett $^{1,50}$. Dongrui $\mathrm{Wu}^{1,51} \cdot$ Karolina Żyniewicz ${ }^{1,52} \cdot$ Nima Rezaei ${ }^{1,2,3}$

$1 \mathrm{http}: / /$ usern.org

2 School of Medicine, Tehran University of Medical Sciences, Tehran, Iran

3 Research Center for Immunodeficiencies, Children's Medical Center Hospital, Tehran University of Medical Sciences, Tehran, Iran

4 Division of Allergy and Immunology, Hospital Nacional Edgardo Rebagliati Martins Lima, Lima, Peru

5 Department of Biology and Health Sciences, College of Science and Health Professions, Edinboro University of Pennsylvania, Edinboro, USA

6 Nutrition and Food Science Area, Preventive Medicine and Public Health, Food Science, Toxicology and Forensic Medicine Department, Faculty of Pharmacy, Universitat de València, Avda. Vicent Andrés Estellés, S/n, València 46100 Burjassot, Spain

7 Department of Applied Science and Technology - DISAT, Politecnico di Torino, Corso Duca degli Abruzzi 24, 10129 Torino, Italy

8 Department of Infectious Diseases, Imperial College London, Norfolk Place, London W2 1PG, UK
9 Physics Department “Ettore Pancini”, University of Naples Federico II Monte Sant'Angelo Campus, via Cinthia 21, 80121 Naples, Italy

10 Institute for Bioengineering of Catalonia (IBEC), Barcelona Institute for Science and Technology, Barcelona 08028, Spain

11 Latvian Institute of Organic Synthesis (LIOS), Riga LV-1006, Latvia

12 Psychotherapist in Private Practice, Canterbury, Kent, England

13 Analytical Laboratory Branch, Scientific Centre of Drug and Medical Technology Expertise after Academician E. Gabrielyan CJSC, Yerevan 0051, Armenia

14 INFN Sezione di Padova, Padova, Italy

15 Penn State Lehigh Valley, 2809 Saucon Valley Road, Center Valley, PA 18034, USA

16 National Health Institutes of Health (NIH) Intramural Research Program (IRP), Bethesda, USA

17 Department of Chemistry \& INSTM, University of Pavia, Via Taramelli 14, Pavia I-27100, Italy 
18 Department of Mechanical Engineering, National University of Singapore, Singapore, Singapore

19 Souk Ahras University, Souk Ahras, Algeria

20 The Institute of Philosophy, University of Szczecin, Ul. Krakowska 71-79, Szczecin 71-004, Poland

21 Translational Research Institute, Woolloongabba, QLD, Australia

22 Department of International Education, Education University of Hong Kong, Hong Kong, China

23 The University of Warwick, Coventry, UK

24 Faculty of Biomedical Sciences, Università della Svizzera italiana (USI) Euler Institute, via G. Buffi 13, CH6900 Lugano, Switzerland

25 Department of Pharmacy, University of Naples "Federico II", Via D. Montesano 49, I-80131 Naples, Italy

26 Academy of Fine Art, Carrara, Italy

27 IRCSS NEUROMED, Pozzilli, Italy

28 Ateneo de Davao University, Davao, Philippines

29 Digestive Disease Research Institute, Tehran University of Medical Sciences, Tehran, Iran

30 Lisbon Accounting and Business School, Instituto Superior de Contabilidade e Administração de Lisboa (ISCAL/IPL), Av. Miguel Bombarda 20, Lisbon 1069-035, Portugal

31 Faculty of Physical Education and Sports Management, Singidunum University, Belgrade, Serbia

32 Department of Internal Medicine and Infectious Diseases, Erasmus Medical Center, Erasmus University, Rotterdam, Netherlands

33 Department of Specific Didactics Faculty of Education, University of Burgos, Burgos 09001, Spain

34 Department of Dermatology and The Yale Cancer Center, Yale School of Medicine, New Haven, CT, USA

35 Mathematics, Nandalal Ghosh B.T. College, Kolkata, West Bengal, India

36 Center for Nanofibers and Nanotechnology, National University of Singapore, Singapore, Singapore
37 Institute for Advanced Sustainability Studies (IASS), Berliner Str. 130, Potsdam 14467, Germany

38 University G.D Annunzio, Chieti Pescara, Italy

39 Medical Virology and Epidemiology of Viral Diseases, University Hospital Tübingen, Tübingen 72076, Germany

40 Institute of Physics, Chemnitz University of Technology, Chemnitz 09107, Germany

41 Division of Cardiothoracic Surgery Department of Surgery The Warren Alpert Medical School, Brown University,

Providence, RI 02903, USA

42 Drug Research Program Division of Pharmaceutical Chemistry and Technology Faculty of Pharmacy, University of Helsinki, FI00014 Helsinki, Finland

43 Department of Philosophy Cultural Studies and Information Activity, Volodymyr Dahl East Ukrainian National University, Severodonetsk, Ukraine

44 University of Maryland, College Park, MD, USA

45 Department of Structural Engineering and Building Materials Faculty of Engineering and Architecture Magnel-Vandepitte Laboratory for Structural Engineering and Building Materials, Ghent University, Tech Lane Ghent Science Park, Campus A, Technologiepark Zwijnaarde 60, B- 9052 Gent, Belgium

46 Department of Religious and Comparative Studies, Adam Mickiewicz University, Poznań, Poland

47 Department of Psychology, University of Miami, PO Box 2481850751, Coral Gables, FL 33124, USA

48 Allergy and Immunology Division, Hospital Nacional Edgardo Rebagliati Martins, Lima, Peru

$49 \mathrm{SimEx} /$ FLOW, Engineering Mechanics, KTH Royal Institute of Technology, Stockholm SE-100 44, Sweden

50 Department of Nutrition, Harvard T.H. Chan School of Public Health, Boston, MA, USA

51 School of Artificial Intelligence and Automation, Huazhong University of Science and Technology, Wuhan 430074, China

52 Artes Liberales Faculty, University of Warsaw, Warsaw, Poland 